\title{
Digital holographic setups for phase object measurements in micro and macro scale
}

\author{
Vít Lédl ${ }^{1, a}$, Pavel Psota ${ }^{1}$, Roman Doleček ${ }^{1}$, Tomáš Vít ${ }^{2}$ \\ ${ }^{I}$ Technical University of Liberec, Institute of New Technologies and Applied Informatics \\ ${ }^{2}$ Technical University of Liberec, Department of Power Engineering Equipment
}

\begin{abstract}
The measurement of properties of so called phase objects is being solved for more than one Century starting probably with schlieren technique 1 . Classical interferometry served as a great measurement tool for several decades and was replaced by holographic interferometry, which disposes with many benefits when compared to classical interferometry. Holographic interferometry undergone an enormous development in last decade when digital holography has been established as a standard technique and most of the drawbacks were solved. The paper deals with scope of the huge applicability of digital holographic interferometry in heat and mass transfer measurement from micro to macro scale and from simple 2D measurement up to complex tomographic techniques. Recently the very complex experimental setups are under development in our labs combining many techniques leading to digital holographic micro tomography methods.
\end{abstract}

\section{Introduction}

Holographic interferometry (HI) is used in measurement of phase objects with great success for more than 40 years. Since its start many different applications have been developed and tested. Recent developments in digital holographic interferometry (DHI) made this technique even more convenient and many experimental difficulties were mitigated. HI as an experimental technique occupies important place in field of heat and mass transfer but in combination with techniques like tomography, microscopy or averaging of hundreds of frames were made feasible after "digital age" arrival. In this paper we would like to show the enormous extent of application where DHI could be used.

\section{DHI principles}

Use In in digital holography, CCD or CMOS sensors are used to record microinterference pattern where the information about the phase of the incoming waves is coded. The microinterference pattern is simply formed by superposition of reference wave $U_{r}$ and an object wave $U_{o}$. The recorded pattern is called the hologram $H$ and is expressed by relation

$$
H \approx\left|U_{o}+U_{r}\right|^{2}=\left|U_{o}\right|^{2}+\left|U_{r}\right|^{2}+U_{o} U_{r}^{*}+U_{r} U_{o}^{*}
$$

To obtain well defined microinterference pattern the waves has to be coherent. Lack of suitable coherent sources has been a major issue after the discovery of holography 2 until first laser was used in holography. The in line setups were use at that pioneering times until Leith and Upatnieks brought the off axis setups 3. In the sixties the very advanced fine grain holographic emulsions Lippmann emulsions were available so the maximum angle between the two interfering waves was not an issue. The first digital holographic approach made by Shnars 4 opened completely new discipline where everything what was standard until then had to be rethought. On the other hand digital holography made things easier especially experiment wise. The maximum angle $\theta_{\max }$ between the two interfering waves is related to spatial frequency, which the given $\mathrm{CCD}$ can record without violating the Nyquist criterion, (it means two pixels for one interference fringe). This condition is expressed by the following relation

$$
\theta_{\max }=2 \arcsin \left(\frac{\lambda}{4 \Delta \xi}\right) \approx \frac{\lambda}{2 \Delta \xi},
$$

where $\Delta \xi \times \Delta \xi$ is the pixel size and $\lambda$ is the wavelength of the used light. The limited spatial frequency which could be recorded is probably the biggest drawback of DHI in comparison to classical holography. Limited spatial frequency is translated to limited angle between the two interfering waves so the size or distance of the object from the detector is influenced.

Digital holography consists of two steps: the recording of the digital hologram and its reconstruction. Digitally recorded hologram is transferred to a computer as an array of numbers. The propagation of optical fields

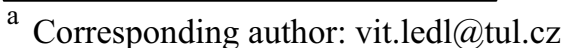


is completely described by diffraction theory, which allows numerical reconstruction of the image as an array of complex numbers representing the amplitude and phase of the optical field. In comparison with classical holography in DHI the reconstruction process is done completely numerically 5 .

Let the phase object be located at a distance $d$ far from the sensor of a digital camera having a coordinates $\xi, \eta$. For digital reconstruction the recorded hologram $H(\xi, \eta)$ is multiplied with a numerical model of the reference wave $r(\xi, \eta)$. For a planar reference wave $r(\xi, \eta)=1$. The complex field in the image plane $U$ is calculated by the Sommerfeld formula which describes the diffraction of a light wave by the hologram grating in distance $d$ from the hologram 6:

$U(x, y)=\frac{1}{j \lambda} \iint H(\xi, \eta) r^{*}(\xi, \eta) \frac{\exp (j k r)}{r} d \xi d \eta$

where

$$
r=\sqrt{d^{2}+(\xi-x)^{2}+(\eta-y)^{2}} .
$$

Coordinates in the image plane are denoted as $x, y$. The Sommerfeld integral is usually solved either by the so called convolution approach or by Fresnel transform. In convolution method the reconstruction formula (3) can be interpreted as a superposition integral:

$$
\begin{aligned}
& U(x, y)= \\
& \quad=\iint H(\xi, \eta) r^{*}(\xi, \eta) g(x-\xi, y-\eta) d \xi d \eta
\end{aligned}
$$

with the impulse response:

$$
g(x, y)=\frac{1}{j \lambda} \frac{\exp \left(j k \sqrt{d^{2}+x^{2}+y^{2}}\right)}{\sqrt{d^{2}+x^{2}+y^{2}}}
$$

The superposition integral can be regarded as a convolution (free space is a linear and shift invariant system) and therefore the convolution theorem

$$
\begin{aligned}
& U(x, y)= \\
& =\mathfrak{I}^{-1}\left\{\mathfrak{I}\left(H(\xi, \eta) r^{*}(\xi, \eta)\right) \mathfrak{I}(g(x, y))\right\}
\end{aligned}
$$

is used. The Fourier transform of $g(x, y)$ can be calculated and expressed analytically as well. This saves one Fourier transform for reconstruction.

In paraxial approximation when the sensor dimensions are negligible in comparison to reconstruction distance $d$ the distance of image point from the hologram plane $r$ defined in (3) can be simplified to:

$$
\begin{aligned}
r & =\sqrt{d^{2}+(\xi-x)^{2}+(\eta-y)^{2}} \approx \\
& \approx d+\frac{1}{2 d}\left[(\xi-x)^{2}+(\eta-y)^{2}\right]
\end{aligned}
$$

and formula (3) becomes:

$$
\begin{aligned}
& U(x, y)=\frac{i}{\lambda d} \exp \left(-i \frac{2 \pi}{\lambda} d\right) \times \\
& \quad \times \exp \left[-i \frac{\pi}{\lambda d}\left(x^{2}+y^{2}\right)\right] \times \\
& \quad \times \int_{-\infty-\infty}^{\infty} \int^{\infty} H(\xi, \eta) r^{*}(\xi, \eta) \exp \left[-i \frac{\pi}{\lambda d}\left(\xi^{2}+\eta^{2}\right)\right] \times \\
& \quad \times \exp \left[i \frac{2 \pi}{\lambda d}(\xi x+\eta y)\right] d \xi d \eta
\end{aligned}
$$

Last term in (9) represents Fourier transform and therefore the equation can be practically solved by:

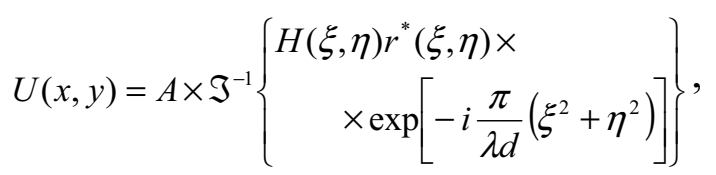

where A is a constant part of (9). All the equations are solved numerically and therefore the both hologram and image plane is sampled. The hologram plane is sampled by pixels having size $\Delta \xi \times \Delta \eta$ and $\Delta x \times \Delta y$ are the image plane pixel sizes:

$$
\begin{aligned}
& \xi=k \Delta \xi \text { where } k \in[1, N], \\
& \eta=l \Delta \eta \text { where } l \in[1, M], \\
& x=n \Delta x \text { where } n \in[1, N], \\
& y=m \Delta y \text { where } m \in[1, M] .
\end{aligned}
$$

In practical applications the integers $\mathrm{N}, \mathrm{M}$ and the pixel size $\Delta \xi \times \Delta \eta$ are defined by the digital camera by default. Image plane distances $\Delta x \times \Delta y$ are given by the size of a frame, where the reconstructed object wave is displayed and depend it also depends on distance $d$ and wavelength $\lambda$ :

$$
\Delta \mathrm{x}=\lambda \mathrm{d} / \mathrm{N} \Delta \xi \quad \text { and } \quad \Delta \mathrm{y}=\lambda \mathrm{d} / \mathrm{M} \Delta \eta
$$

The formula (10) after sampling becomes:

$$
\begin{aligned}
& U(n \Delta x, m \Delta y)= \\
& \quad=\mathfrak{S}^{-1}\left\{\begin{array}{l}
H(n \Delta \xi, m \Delta \eta) r^{*}(n \Delta \xi, m \Delta \eta) \times \\
\times \exp \left[-i \frac{\pi}{\lambda d}\left((n \Delta \xi)^{2}+(m \Delta \eta)^{2}\right)\right]
\end{array}\right\}
\end{aligned}
$$

In the same manner impulse response (6) is sampled leading to discrete version:

$$
g(k, l)=\frac{1}{j \lambda} \frac{\exp \left(j k \sqrt{d^{2}+(k \Delta \xi)^{2}+(l \Delta \eta)^{2}}\right)}{\sqrt{d^{2}+(k \Delta \xi)^{2}+(l \Delta \eta)^{2}}}
$$

The pixel distances of the images reconstructed by the convolution approach are equal to that of the hologram plane: 


$$
\Delta \mathrm{x}=\Delta \xi \quad \text { and } \quad \Delta \mathrm{y}=\Delta \eta
$$

The pixel distances of the reconstructed images by convolution method (16) differ from those of the Fresnel approximation (13). At first sight it seems to be possible to achieve a higher resolution with the convolution approach if the pixel distance is small enough. On closer examination one recognizes that the resolution calculated by (16) is only a numerical value. The physical image resolution is determined by diffraction limit. Considering the digital camera sensor with dimensions $\mathrm{N} \Delta \xi \times \mathrm{M} \Delta \eta$ as an aperture of the optical system, the diffraction limit (Airy disc diameter) defined by this rectangle aperture corresponds to the resolution of the Fresnel transformation (13). It follows that Fresnel transformation can be regarded as the 'automatic scaling' algorithm, setting the resolution of the image reconstructed by a discrete Fresnel transform always to the physical limit.

One should note, the both Fresnel transformation and convolution method can be effectively calculated with the use of FFT algorithm (Fast Fourier Transform).

The reconstructed wave field includes information about the object wave (real image) as well as about its conjugated wave (virtual image) and reference wave (DC term). This is the result of the reconstruction interference equation (1). The real image corresponds to a wavefront converging to a sharp image, while the virtual image belongs to a divergent wavefront that seems not to be in focus. Using formula

$$
\begin{aligned}
H(\xi, \eta) & =\left(H_{1}(\xi, \eta)-H_{3}(\xi, \eta)\right)+ \\
& +j\left(H_{4}(\xi, \eta)-H_{2}(\xi, \eta)\right)
\end{aligned}
$$

where $H_{1} \ldots H_{4}$ are $\pi / 2$ phase shifted holograms and $j=\sqrt{-1}$, the resulting complex field $H$ in hologram plane could be calculated 7 . The benefit of phase shifting is that the complex field $H$ is free of undesired diffraction orders (DC term and virtual image) and the obtained phase we get with higher precision. Otherwise the unwanted diffraction orders could overlap the desired information in hologram spectrum and decrease the SNR in real image. Therefore the reconstructed real image of object can fulfil the whole reconstructed field and better lateral resolution is reached.

In HI one of the waves has to be reflected from or transmitted through the object undergoing the measurement. Usually it is the object wave which interacts with the object. Heat and mass transfer problems are specific by the fact that the object wave propagates through the transparent media and its amplitude is not significantly changed during the interaction but the phase is significantly affected, or affected at least so it could be detected. In the classical double exposure method of holographic interferometry two wavefronts influenced by the same object (e.g. initial state and a state after a temperature change) are recorded consecutively onto the same holographic plate. In fact HI can bring to interference two or more wave fields simultaneously which existed at different times. After the reconstruction by illumination the hologram with the laser light, the both reconstructed waves are superposed, which leads to resulting interference pattern reflecting the phase difference $\Delta \varphi$ between the two object states. The phase difference bears the information about the phase object variations and can be converted to usually desired quantities like temperature, refractive index or pressure variations.

In the digital HI the principle is similar except the superposition of the two waves is performed numerically. For that at least two digital holograms $H_{1}, H_{2}$ are captured. The first hologram corresponds to an initial (reference) state of phase field and the second hologram is captured when the temperature field has been varied. Two wave fields are reconstructed from the digital holograms can be defined as:

$$
\begin{aligned}
U_{1}(n \Delta x, m \Delta y)= & \left|U_{1}(n \Delta x, m \Delta y)\right| \times \\
& \times \exp \left[\varphi_{1}(n \Delta x, m \Delta y)\right] \\
U_{2}(n \Delta x, m \Delta y)= & \left|U_{2}(n \Delta x, m \Delta y)\right| \times \\
& \times \exp \left[\varphi_{2}(n \Delta x, m \Delta y)\right]
\end{aligned} .
$$

When the wave fields are divided one by another $U_{1} / U_{2}$, their phase difference $\Delta \varphi$ is subtracted, and it can be shown that phase difference $\Delta \varphi$ can be directly calculated by

$$
\Delta \varphi=\arg \left(\frac{\operatorname{Im}\left\{U_{1}\right\} \operatorname{Re}\left\{U_{2}\right\}-\operatorname{Im}\left\{U_{2}\right\} \operatorname{Re}\left\{U_{1}\right\}}{\operatorname{Im}\left\{U_{1}\right\} \operatorname{Im}\left\{U_{2}\right\}+\operatorname{Re}\left\{U_{1}\right\} \operatorname{Re}\left\{U_{1}\right\}}\right) .
$$

The possibility of direct access to the phase difference information is one of the major advantages of DHI over the classical HI, where the phase difference must be evaluated by interference pattern analysis.

\section{Temperature field determination}

Let's assume the first hologram is recorded with refractive index $n_{1}$ and the second hologram with $n_{2}$. The relation between refractive index change $\Delta n=n_{2}-n_{1}$ and interference phase is given by

$$
\Delta \varphi=\frac{2 \pi}{\lambda} \oint_{L}\left[\Delta n-n_{\infty}\right] d l
$$

where $d l$ denotes the differential distance along the line $L$, $n_{\infty}$ is refractive index of surrounding air. The solution of (20) depends on the type of refractive index field distribution. In practice we can divide the refractive index fields into three groups: flat, symmetric and general (asymmetric) fields.

\subsection{Flat or symmetric (2D) refractive index fields}

The easiest case of refractive index field is the $t$ refractive index field varying in only one direction. Since there is no refractive index variation along the object length $\mathrm{L}$, 
formula for the interference phase calculation (20) becomes:

$$
\Delta \varphi=\frac{2 \pi}{\lambda} L\left[\Delta n-n_{\infty}\right]
$$

In axially-symmetric temperature fields, the refractive index (or temperature distribution) is a function of radius $r$ and formula (20) becomes:

$$
\Delta \varphi=\frac{4 \pi}{\lambda} \int_{-\infty}^{\infty} \frac{r\left[\Delta n-n_{\infty}\right]}{\sqrt{r^{2}-x^{2}}} d r
$$

For the reconstruction of rotationally symmetric temperature field one can use inversion of Abel transform corresponding to the right-hand side of Eq. (22).

\subsection{Asymmetric (3D) refractive index fields}

If asymmetric refractive index field $n(\xi, z)$ is considered we can write the function in polar coordinates $(t, \Phi)$ as following

$$
n(\xi, z)=n(t \cos \Phi, t \sin \Phi) .
$$

The determination of asymmetric fields requires the analysis of a large number of holographic interferograms by methods of tomography. In this case the line integral transform defined in (20) is mathematically equivalent to Radon Transform 8:

$$
g(s, \theta)=\iint n(\xi, z) \delta(\xi \cos \theta+z \sin \theta-s) d \xi d z
$$

\section{(24)}

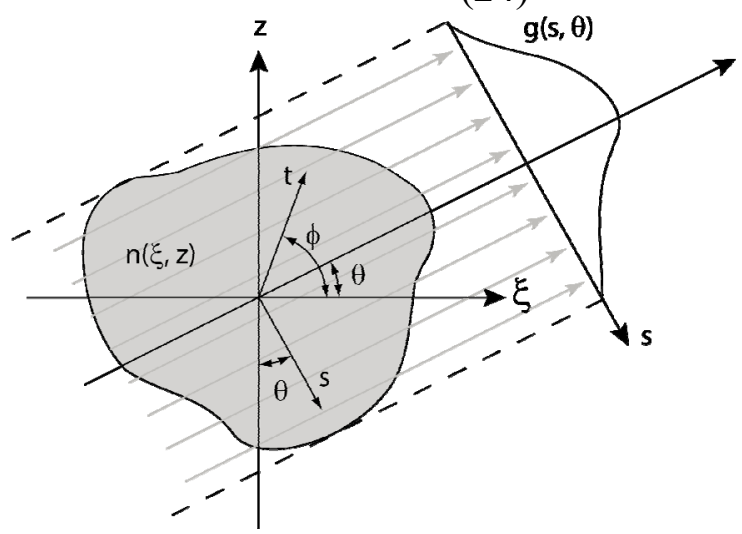

Figure 2. The recording of asymmetric refractive index fields

where $n(\xi, z)$ is the refractive index distribution in Cartesian coordinates, $\delta$ is the Dirac pulse, $\theta$ is the angle of projection and $s$ is the shift distance from the centre. The data obtained by Radon Transform $g(s, \theta)$ is called sinogram. The name sinogram originated because the Radon transform of a Dirac delta function is a distribution supported on the graph of a sine wave.

In practice, the sinogram is a $2 \mathrm{D}$ array containing the projections from different angles in columns of the array.
Reconstruction is based on back-projection mathematically described by Inverse Radon Transform 9:

$$
n(\xi, z)=\int_{0}^{\pi} g(\xi \cos \theta+z \sin \theta, \theta) d \theta
$$

The back-projection operation propagates the measured sinogram back into the image space along the projection paths. Further, images for all rows are stacked to get $3 \mathrm{D}$ volume data.

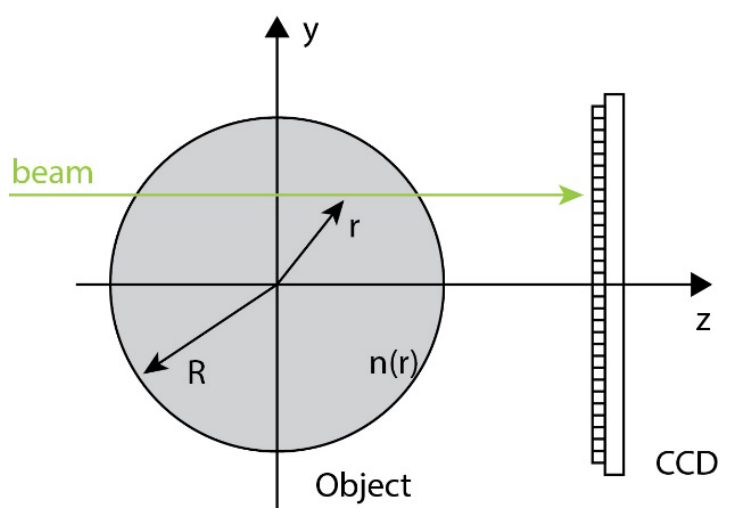

Figure 1. The recording of rotationally symmetric refractive index field.

\subsection{Temperature field calculation}

The temperature field and refractive index field are related by ideal gas equation and Gladston-Dale equation:

$$
n-1=K . \rho,
$$

where $K$ is Gladston-Dale constant which is property of gas. The Gladston-Dale constant is almost independent from pressure or temperature under the moderate physical conditions. It is slightly dependent on wavelength of the light. The density $\rho$ of the gas can be calculated from the pressure $P$, the molecular weight $M$ and absolute temperature $T$ via the ideal gas equation:

$$
\rho=\frac{M P}{R T}
$$

combined with the universal gas constant, $R=8,3143 J K^{-1} \mathrm{~mol}^{-1}$. Formula (27) yields with (26)

$$
n-1=\frac{K M P}{R T}
$$

Holographic interferometry has two major benefits in comparison to classical interferometry. The first one is its ability to measure diffusively reflecting surfaces, which is not the subject of phase object measurement, so we are not going to discuss it further in text. The second benefit is the fact that the technique is differential. In the 
classical interferometry both arms of the interferometer has to be equal optically so the minimal difference between the optical paths in every point has to be introduced. The resulting interferogram is combination of measured deviation and the deviation introduced by the interferometer. Unfortunately those two additions are not distinguishable. Therefore the interferometer used for classical interferometry has to be build up from the very high quality optical components and has to be adjusted with very high precision. In the case of holographic interferometer, first the reference state is recorded and then the reference state is compared to state of the measured object undergoing the change. It means that the influence of the unequal paths is diminished and the result of HI or DHI is just the change of those two states. Many holographic setups differing in size or complexity have been developed during the past few decades, serving to broad range of applications. Most of them are outcoming from two basic types of interferometers the Mach-Zehnder and the Twymann-Green.

\section{Experimental Setups}

\subsection{Single paths holographic interferometer}

The most common type of holographic interferometer used in phase object measurement is probably simple Mach-Zehnder type and its modifications. In figure 3 the schematics showing its principle 10 is displayed. Laser beam emitted from laser head is divided by polarizing beam splitter $\mathrm{BS}_{1}$ which could be equipped with half wave plates. Half-wave plates are used to adjust intensity and polarization of beams in each arm of interferometer. Both beams are further filtered by spatial filters SF and collimated. Collimated beam no. 2 is directed to a nonpolarizing beam splitterBS $\mathrm{S}_{2}$. One part of light-wave passes directly through the $\mathrm{BS}_{2}$ and reaches screen $\mathrm{S}$ where the beam is absorbed. Second part is reflected in the $\mathrm{BS}_{2}$ to objective $\mathrm{O}$ and strikes $\mathrm{CCD}$ chip of the camera. Collimated beam no. 2 goes through measured phase-object and is reflected by mirror $\mathrm{M}_{2}$ to beamsplitterBS $\mathrm{S}_{2}$. Again, one part of this beam part hits the screen $\mathrm{S}$ and second one reaches objective $\mathrm{O}$ and CCD chip. Both waves that are impinging on the CCD chip interfere there. At least two digital holograms capturing phase-object in two different physical states are recorded and further numerically processed in the way described previously. Usually first hologram corresponds to reference state and the other is the hologram after the change to the object happened. The area of interest is limited by the size of the beamsplitter $\mathrm{BS}_{2}$ and the objectives usually it is not bigger than 50 by $50 \mathrm{~mm}$.

Figure 2 shows the reconstructed interference phase distribution and corresponding temperature fields of the resistor and resistive spiral heated by the electric current. Fields are the projections of the temperature changes in direction of the object beam. The main advantage of this interferometric setup is its relative simplicity. The adjustment requires experienced person. The sensitivity of the interferometer is low, on the other hand this feature is welcomed if huge refractive index changes are investigated.

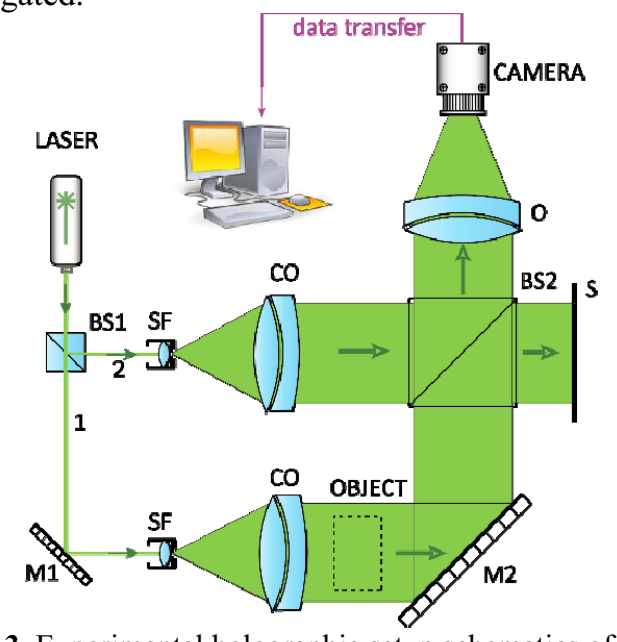

Figure 3. Experimental holographic setup schematics of MachZehnder type of interferometer. BS1-polarizing beam splitter, SF-spatial filter, CO-collimation objective, BS2-nonpolarizing bemasplitter, O-objective lens, M-mirror, S-screen.

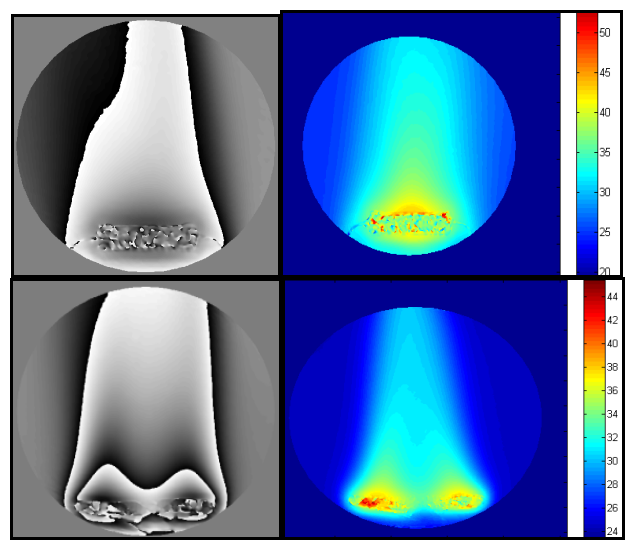

Figure 4. Reconstructed interference phase and corresponded temperature field measured on Mach-Zehnder interferometer.

\subsection{Double path Holographic interferometer}

For the measurement of faint phase changes the sensitivity of Mach-Zehnder type of interferometer is insufficient. Altered setup based on Twymann-Green interferometer offers twice the sensitivity compared to Mach-Zehnder type of interferometer. Working principle of such interferometer is shown in figure 3. The laser beam is divided by polarizing beam splitter $\mathrm{BS}_{1}$ and two new originated beams are modified similarly as it was in the previous setup. Beam no. 1 is reflected by mirror $\mathrm{M}_{2}$ to beam splitter $\mathrm{BS}_{2}$. One part of the beam no. 1 goes directly through beamsplitter and objective $\mathrm{O}$ to $\mathrm{CCD}$ chip. Second part is reflected to collimation objective CO. Collimated beam no. 2 enters the beam splitter $\mathrm{BS}_{2}$. One part of the beam no. 2 is reflected in direction to $\mathrm{M}_{2}$, second part passes through the measured object. Mirror $\mathrm{M}_{3}$ is placed behind the object and it reflects the beam back through the object to the beam splitter $\mathrm{BS}_{2}$. There the beam undergoes second division and part of it is directed through the objective $\mathrm{O}$ to the CCD chip. 


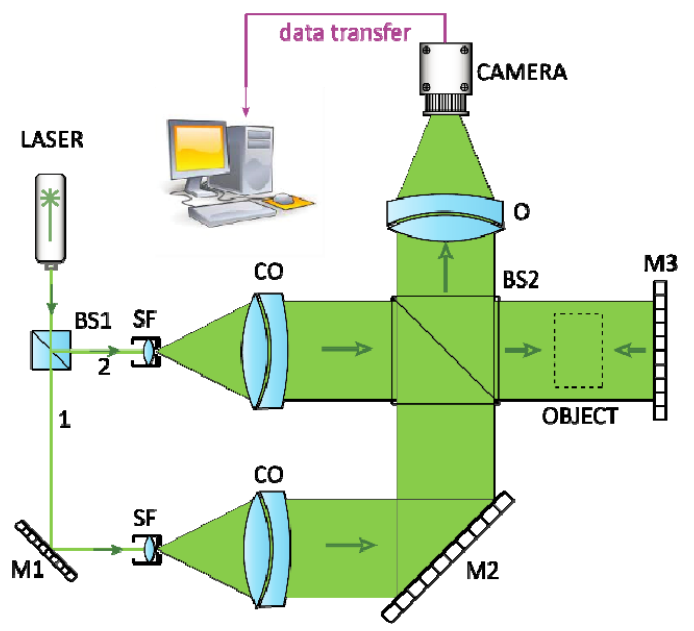

Figure 5. Experimental holographic setup schematics of Michelson type of interferometer with double pass. BS1polarizing beam splitter, SF-spatial filter, CO-collimation objective, BS2-nonpolarizing bemasplitter, O-objective lens, Mmirror.

This setup forces the beam to pass through the measured object twice which results in higher sensitivity. It can be seen in double fringe-count in reconstructed interference phase (see figure 6). On the other hand such setup is more complicated and difficult to adjust to right performance. The object should be placed as close to the mirror $M_{3}$ as possible in order to guarantee that rays are following the same paths in the phase object.

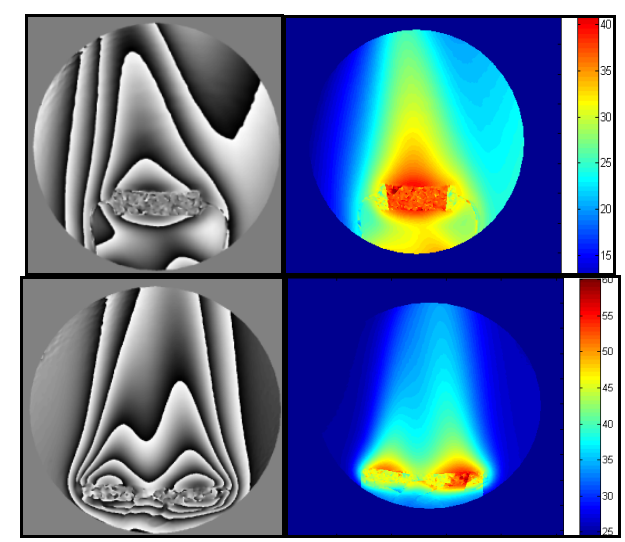

Figure 6. Reconstructed interference phase and corresponded temperature field measured on Twymann - Green type of interferometer.

There is also higher energy loss on the divider $\mathrm{BS}_{2}$. Therefore using this setup longer exposure time is required for hologram recording in comparison with Mach-Zehnder type of interferometer. In the measurement of the fast varying refractive index fields the long capture time can cause low contrast or microinterference pattern (hologram) blur. To address previously mentioned problem with light efficiency, there are several possible solutions depending on the problem under investigation. Higher power laser would be probably the straightforward one, than there is the use of pulse laser with synchronized camera or with recent developments the more sensitive high speed cameras are available.

\subsection{Large area measurement}

As it was mentioned previously the size of the measurement area is given by the size of optical components used in the setup mainly the size of the beam splitter $\mathrm{BS}_{2}$ and the collimating lenses. For wide area measurement the proper beam expander is inevitable in the setup with double path. Double paths setup brings the benefit of double sensitivity but on the other hand any wavefront distortions caused by imperfections in the optical system of expander are magnified as well. The expander imperfections could origin either from improper assembly of expander or more frequently from nonoptimized design. The working principle of the interferometer with expander is shown in figure 7.

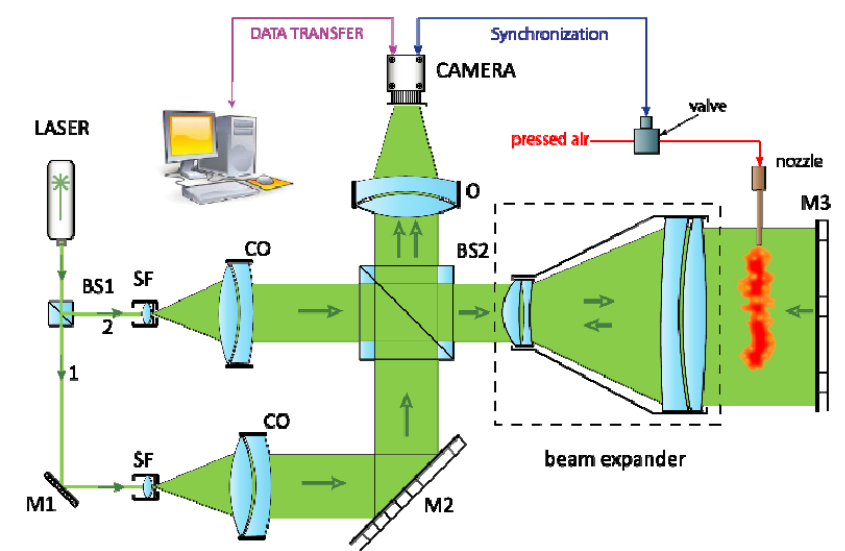

Figure 7. DHI setup with double path and with beam expander.

The shown expander is based on modified standard telescope design and by optimization of two doublets one can get an aplanatic system of the expander. The output diameter of the beam is $120 \mathrm{~mm}$. The function of the beam expander is demonstrated by the experiment of the measurement of the pressed air puff from the weaving nozzle (see figure 8). The high speed camera (5000FPS) was used for capturing holograms of this fast developing pressure field.

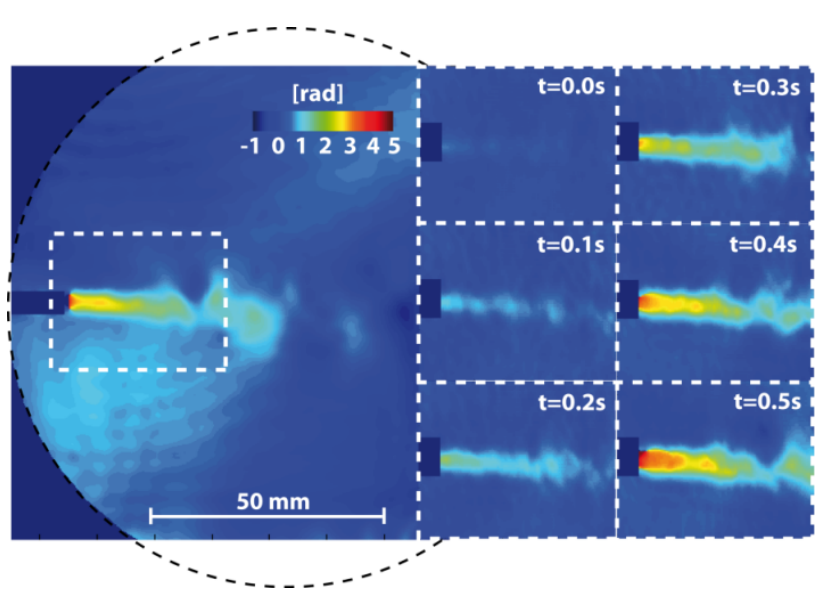

Figure 8. The phase distribution and development in the time of the pressured air puff. 


\subsection{Coherent phenomena measurement}

For measurement of the fast periodical refractive index field development it is not necessary to use always the high speed camera. One can consider this phenomenon as coherent and synchronization of the phenomenon phase with the camera capture time can be applied with success. The external trigger of the camera enables to capture the hologram with a precisely defined delay. Since the synchronization frequency is higher than the camera frame rate, only some of the pictures are selected from the whole series. The hologram is not captured after each synchronization signal but some periods of the phenomenon are skipped (see figure 9). Therefore it is possible to measure very high frequency phenomena with use of relatively low frame rate camera 11 .

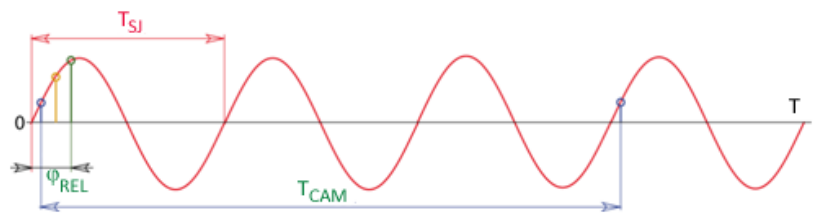

Figure 9. Synchronization and triggering between the camera and phenomenon. TSJ - period of the phenomenon, $\varphi$ REL relative phase of the phenomenon (defined delay), TCAM capture time of the camera

For demonstration we selected the synthetic jet SJ 12. SJ are jets of fluid that are generated by pushing or pulling a fluid through an orifice by the interactions within the train of counter-rotating vortex pairs. SJ are generated with frequency $15 \mathrm{~Hz}$ by one pair of blowers. Although we used double sensitivity interferometer the temperature changes were too low to cause any significant change of refractive index field. Therefore we placed a heating cartridge in the space in between the blowers that heats up the air of the flux of air flowing from the orifice. The heating cartridge was controlled by a connected PID regulator. The principle schema is showed in figure 10.

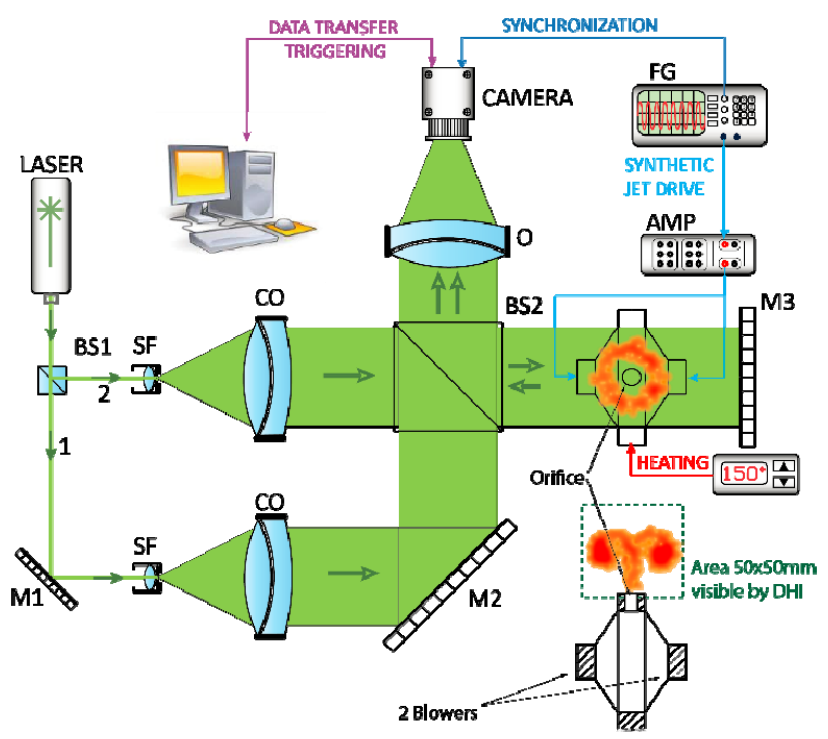

Figure 10. Principle schema of the interferometer with synchronization of the camera and driver of temperature field. $\mathrm{FG}$ - functions generator, AMP - amplifier.
In figure 11 the sequence of the several exposures of a coherent SJ phenomenon is shown. It can be clearly seen that the temperature field for each exposure is almost perfectly similar to the other ones. The reconstructed fields correspond to the same relative phase of phenomenon. The lower row images are standard deviation of each pixel divided by its own value to get a percentage scale. It can be seen that the differences between the fields are less than $5 \%$. This uncertainty is caused by the random fluctuation in the temperature field. However this variance can be reduced by averaging of all fields which are corresponding to the same relative time. The temperature field dynamic evolution in time is shown in figure 12. The holograms were captured at different time delays $-2 \mathrm{~ms}, 8 \mathrm{~ms}$ and $16 \mathrm{~ms}$ counted from the start (zero phase) of the phenomenon.

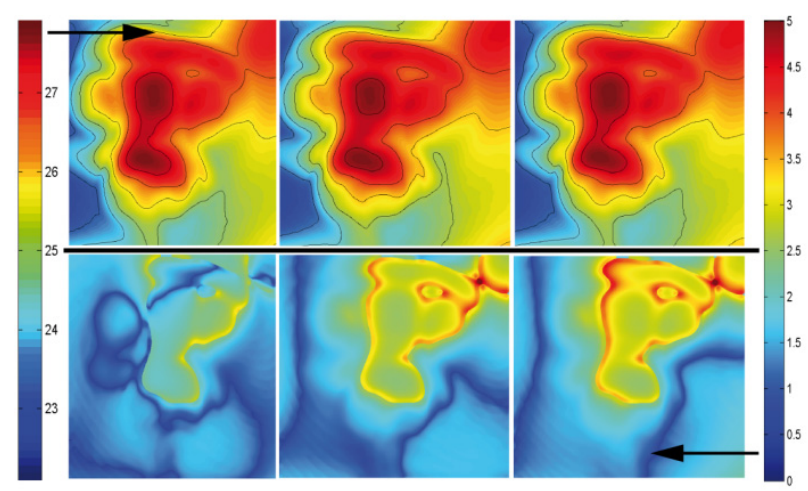

Figure 11. The consequence of a precise camera and SJ driver synchronization. First row shows temperature field $\left({ }^{\circ} \mathrm{C}\right)$ captured at the same time delay $16 \mathrm{~ms}$ in different cycles of the periodical phenomenon. The bottom row displays the standard deviations [\%] of temperature field distributions.

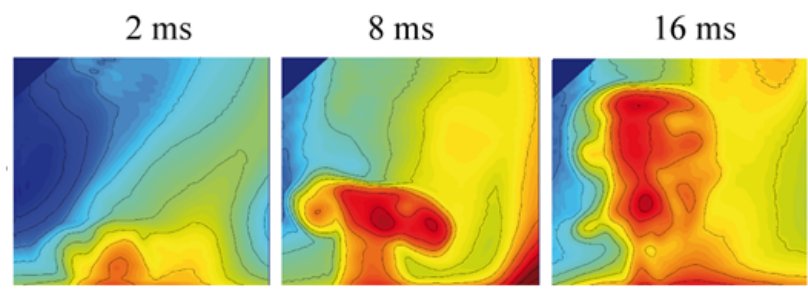

Figure 12. The dynamic evaluation of temperature field $\left({ }^{\circ} \mathrm{C}\right)$.

\subsection{Tomography based reconstruction}

In the simplest case temperature fields are twodimensional or symmetrical and thus could be easily measured and results evaluated. Measurement of asymmetrical temperature fields is more demanding. Sufficient examination of asymmetrical temperature fields cannot be done without the application of a tomographic approach. Tomography generally requires to capture a large number of different projections. In case of DHI it would be necessary to use many digital sensors in the measurement setup to obtain digital holograms for different projection directions. This would make the holographic setup very complex and expensive and the adjusting would be real nightmare. However as it was shown in the previous chapter there could be smarter way proposed - at least for the coherent phenomenon 
measurement. With use of a rotation stage one can capture the area of interest from different directions in precisely defined relative phase of the phenomenon [13].

The principle of experimental setup function is shown in figure13. Object under investigation is SJ. The generator of SJ (cavity and pairs of blowers) is placed on the rotation stage. In the first step the sequence of digital holograms for defined relative delays is captured (whole phenomenon period). One can capture number of holograms for each delay for the subsequent averaging. In the second step the rotation stage is turned at the specific angle and again is captured sequence of holograms. This process is repeated for sufficient number of angles. The interference phase distributions are computed for all holograms. Then is preformed backprojection (12) and all rows of the 2D back-projected field are stacked to get 3D volume data. The final result $3 \mathrm{D}$ volume data of the temperature field are displayed in figure 14 .

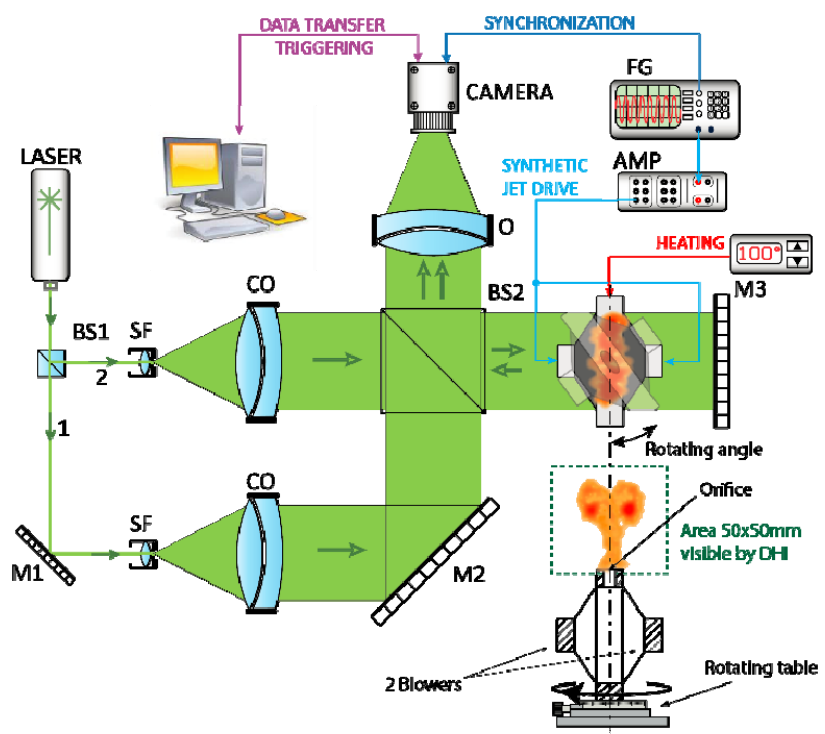

Figure 13. Principle schema of the interferometer with synchronization of the camera and driver of temperature field. $\mathrm{FG}$ - functions generator, AMP - amplifier.

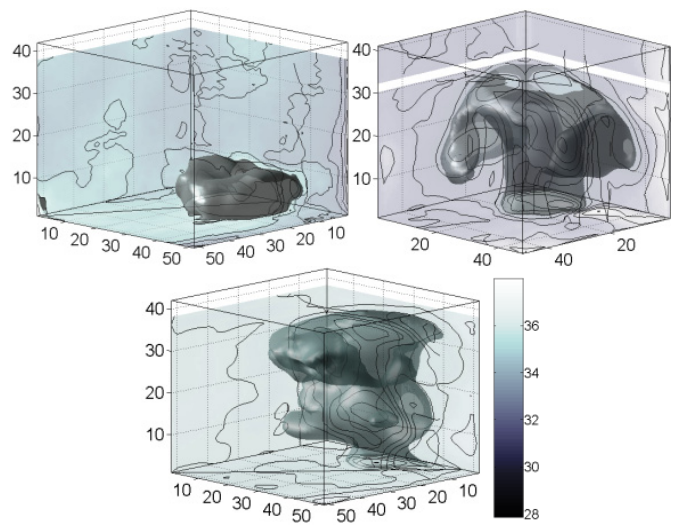

Figure 14. The 3D volume data of the computed temperature field $\left[{ }^{\circ} \mathrm{C}\right]$ of relative delay time $3,2 \mathrm{~ms}, 9,6 \mathrm{~ms}$ and $19,2 \mathrm{~ms}$.

\subsection{Digital holographic microscopy}

Transparent objects, like living biological cells are very often studied by digital holographic microscopy in transmission mode. The main benefit DHM brings is the quantified phase information due to phase shifting principle which can be easily added to DHM setup. The phase shift image is unique for digital holographic microscopy and gives quantifiable information about optical distance. In reflection DHM, the phase shift image forms a topography image of the object. The availability of the phase is standard feature of digital holographic interferometry. In the heat and mass transfer the lateral optical resolution of digital holographic microscopy is important. The lateral resolution of DHM is comparable to the resolution of traditional light microscopy so 0,5 $\mathrm{mm}$ is more less the limit however the axial (depth) resolution is superb and few $\mathrm{nm}$ can be reached. In biology where the resolution is very important microscope objectives are used to image the selected area of the sample to the sensor with proper magnification. In heat and mass transfer it may be replaced by a simple lens if a slightly lower optical resolution is acceptable. On the other hand the microscope objective even the very cheap one is perfectly corrected for single wavelength. Laser beam emitted from laser head is divided by polarizing beam splitter $\mathrm{BS}_{1}$ which could be equipped with half wave plates. Half-wave plates are used to adjust intensity and polarization of beams in each arm of interferometer. Both beams are further filtered by spatial filters SF. Collimated beam no. 2 is directed to a nonpolarizing beam splitter $\mathrm{BS}_{2}$. One part of light-wave passes directly through the $\mathrm{BS}_{2}$ and reaches screen $\mathrm{S}$ where the beam is absorbed. Second part is reflected in the $\mathrm{BS}_{2}$ to objective $\mathrm{O}$ and strikes $\mathrm{CCD}$ chip of the camera. The wave going through the object is collected by a microscope objective e.g. infinity corrected one and enters the $\mathrm{BS}_{2}$, the object and reference wave fronts are joined by a beam splitter to interfere and create the microinterference pattern (hologram) which is recorded by CCD or CMOS sensor. Numerical reconstruction algorithm is than applied on phase shifted data. The heterodyne frequency of the two synchronously operating Bragg cells is set to be four times bigger than the camera frame rate. So every of four consequent images taken by the camera creates the $\pi / 2$ phase shifted data.

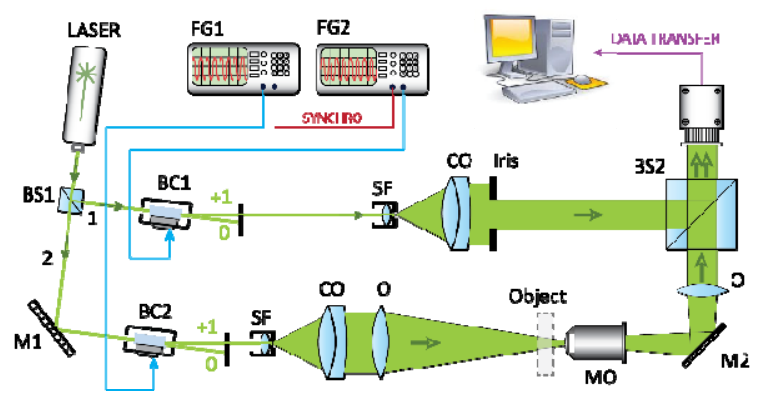

Figure 15. Principle schematics of digital holographic microscopy with phase shifting done by Bragg cells operating in heterodyne mode 


\section{References}

1. M. Giglio, S. Musazzi, and U. Perini, Opt. Commun., $36(1981)$

2. D. Gabor, Nature, 161 (1948).

3. E. N. Leith and J. Upatnieks, Journ. Opt. Soc. Amer., 54 (1964).

4. L. P. Yaroslavskii and N. S. Merzlyakov, Methods of Digital Holography (Consultants Bureau, New York, 1980)

5. T. H. Demetrakopoulos and R. Mittra, Appl. Opt., 13 (1974)

6. T. Kreis, Handbook of Holographic Interferometry: Optical and Digital Methods (Wiley-VCH, 2005)

7. I. Yamaguchi, T. Zhang, Opt. Lett., 22 (1997).

8. S.R. Deans, The Radon Transform and Some of Its Applications (New York, 1983)

9. S. R. Deans, The Radon Transform and Some of Its Applications (Dover, 2007).

10. V. Lédl, T. Vít, R. Doleček, P. Psota, EPJ Web of Conferences, 25 (2012)

11. P. Psota, V. Lédl, R. Doleček, T. Vít, J. Václavík, Optical Measurement Techniques for Systems \& Structures $^{2}$, (Shaker, Aachen, p331-338, 2012)

12. Trávníček, Tesař, Int. J. Heat Mass Transfer, 46 (2003)

13. R. Doleček, P. Psota, V. Lédl, T. Vít, J. Václavík, V. Kopecký, Applied Optics, 52 (2013)

\section{Acknowledgement}

We gratefully acknowledge the support of the Grant Agency of the Czech Republic - Czech Science Foundation (project no. 14-08888S). 